ISAHP 2005, Honolulu, Hawaii, July 8-10, 2005

\title{
A method to speedily pairwise compare in AHP and ANP
}

\author{
Kazutomo Nishizawa \\ Department of Mathematical Information Engineering, \\ College of Industrial Technology, Nihon University \\ 1-2-1 Izumi-cho, Narashino, Chiba 275-8575, Japan \\ k7nisiza@cit.nihon-u.ac.jp
}

Keywords: Speedily pairwise comparisons, 2-cluster ANP, Binary AHP

Summary: AHP (Analytic Hierarchy Process) and ANP (Analytic Network Process) are useful tool for decision makers. However, the amount of pairwise comparison becomes large with increasing the number of alternatives and criteria. Therefore, it takes much time and the loads of the decision maker increase. In AHP and ANP, it is important to pairwise compare carefully but to need speedily. This paper proposes a method to deal speedily with pairwise comparisons, and apply our method to AHP and 2cluster ANP. In our method, we introduce three-level evaluation, scale values range from 0 to 2. At first, decision maker sets the standard in each criterion, and next, we evaluate alternatives and construct the matrix, called evaluation matrix. In AHP, based on evaluation matrix, we construct comparison matrix automatically and have each weight of alternative. In 2-cluster ANP, equivalent to AHP, we construct super matrix automatically and have each weight. The usefulness of our method was confirmed through some examples.

\section{Introduction}

AHP (Analytic Hierarchy Process) (Saaty, 1980) and ANP (Analytic Network Process) (Saaty, 2001) are useful tool for decision makers. However, the amount of pairwise comparison becomes large with increasing the number of alternatives and criteria. Therefore, it takes much time and the loads of the decision maker increase.

In AHP and ANP, it is important to pairwise compare carefully but to need speedily. To implement AHP in decision-making part of several software, it is indispensable to construct comparison matrix and super matrix speedily and automatically. This paper proposes a method to deal speedily with pairwise comparisons, and apply our method to AHP and 2-cluster ANP.

In our method, we introduce three-level evaluation, scale values range from 0 to 2. At first, decision maker sets the standard in each criterion, and next, we evaluate alternatives and construct the matrix, called evaluation matrix. For each criterion, if alternative satisfy the standard then let evaluation value be 2 , else be 0 , but unknown be 1 .

In AHP, based on evaluation matrix, we construct comparison matrix automatically and have each weight of alternative. In 2-cluster ANP, equivalent to AHP, we construct super matrix automatically and have each weight. To determine these weights, in our method, we introduce binary AHP (Takahashi, 1990).

In this paper, the procedure of speedily pairwise comparison is described in section 2. In section 3, illustrates an example by proposed methods. Finally conclude in this investigation in section 4 . 


\section{Procedure}

In this section, the procedure of speedily pairwise comparison is proposed. Assume decision maker prioritize $n$ alternatives based on $m$ criteria.

At first, decision maker selects $m$ criteria, and sets the standard in each criterion carefully and next evaluate alternatives. In our method, we introduce three-level evaluation, scale values range from 0 to 2. The details are shown in Table 1.

\begin{tabular}{cc}
\multicolumn{2}{c}{ Table 1 Three-level evaluation } \\
Standard of & $\begin{array}{c}\text { Evaluation } \\
\text { Value }\end{array}$ \\
Criterion & 2 \\
satisfy & 0 \\
not satisfy & 1 \\
unknown &
\end{tabular}

Next, to prepare for our method, we construct the matrix $D$, called evaluation matrix. We denote the element of the matrix $D$ by $d_{i j}$ ( $i=1$ to $m$ and $j=1$ to $n$ ). For example, if alternative $a_{j}$ satisfy the standard of criterion $c_{i}$ then let be $d_{i j}=2$ else be $d_{i j}=0$, but unknown be $d_{i j}=1$.

Based on matrix $D$, we describe our method to speedily construct comparison matrix and super matrix, respectively.

\subsection{Procedure in AHP}

In AHP, we denote the weights of alternatives by $w$ and calculate by following equation.

$$
w=W V
$$

As well known the matrix $W$ consists of the column vector $W c_{i}(i=1$ to $m)$, as follows:

$$
W=\left[\begin{array}{llll}
W c_{1} & W c_{2} & \cdots & W c_{m}
\end{array}\right] .
$$

$W c_{i}$ is the weight of alternatives with respect to criterion $c_{i}$, from comparison matrix $A c_{i}$.

To construct comparison matrix, based on $D$, we introduce binary comparison. In our method, we denote the element of comparison matrix $A c_{i}$ by $a_{x y}(x, y=1$ to $n)$ and $a_{x y}=\theta\left(d_{i x}>d_{i y}\right), a_{y x}=1 / a_{x y}, a_{x x}=1$ and $\theta(>1)$ is a parameter.

To construct $W$, proposed procedure is summarized, as follows:

(W-0): $i=0$.

(W-1): Add 1 to $i$.

(W-2): Pairwise binary compare with $d_{i j}$, ith-row vector of $D$, for $j=1$ to $n$.

(W-3): Construct comparison Matrix $A c_{i}$.

(W-4): Calculate $W c_{i}$ from $A c_{i}$.

(W-5): Repeat (W-1) to (W-4) until $i=m$, and construct $W$.

Next, we construct $V$ speedily. The vector $V$ is the weights of criteria. To calculate $V$, in general, there are two kind of method. One is by ordinary pairwise comparison between criteria and the other is by calculate the weights automatically. In this paper, we introduce the latter method. 
To prioritize criteria and have corresponding weights, in this paper, we use the frequency of evaluation value 2 (satisfied) on $D$. Furthermore there are two kind of concept, one is give a high priority to frequent of satisfy criterion and the other is give a high priority to infrequent criterion. There are various methods to decide the weights, for example by binary AHP and so on.

Finally, we can obtain $W$ and $V$, above procedure, and have $w$ from (1).

\subsection{Procedure in 2-cluster-ANP}

In 2-cluster ANP, equivalent to AHP, we construct super matrix $S$ automatically and have each weight. Super matrix $S$ of 2-cluster ANP consists of sub matrix $W, V$, and $O$, as follows:

$$
S=\left[\begin{array}{l|l}
O & V \\
\hline W & O
\end{array}\right] .
$$

From (3), we have the weights of alternatives $w$ and the weights of criteria $v$.

In (3), $O$ is the zero matrix. The sub matrix $W$ is coinciding with (2). The sub matrix $V$ consists of the column vector $V a_{j}(j=1$ to $n)$, as follows:

$$
V=\left[\begin{array}{llll}
V a_{1} & V a_{2} & \cdots & V a_{n}
\end{array}\right] .
$$

$V a_{j}$ is the weight of criterion for alternative $a_{j}$, from comparison matrix $A a_{j}$.

If alternative $a_{j}$ satisfy the standard of criterion $c_{x}\left(d_{x j}=2\right)$ and not satisfy the standard of criterion $c_{y}$ $\left(d_{y j}=0\right)$, then we judge that $c_{x}$ is more favor than $c_{y}$ for $a_{j}$. So to construct $A a_{j}$, we use $j$ th-column vector of $D$.

To construct $V$, proposed procedure is summarized as follows:

$(\mathrm{V}-0): j=0$.

(V-1): Add 1 to $j$.

(V-2): Pairwise binary compare with $d_{i j}$, jth-column vector of $D$, for $i=1$ to $m$.

(V-3): Construct comparison Matrix $A a_{j}$.

(V-4): Calculate $V a_{j}$ from $A a_{j}$.

(V-5): Repeat (V-1) to (V-4) until $j=n$, and construct $V$.

From (3), we have the weights of alternatives $w$, and the weights of criteria $v$, simultaneously. To calculate $w$ and $v$ from super matrix, there are two kind of method. One is calculating the infinite power of $S$ and the other is calculating the principle eigen vector corresponding to the eigen value 1 . In this study, we calculate $w$ and $v$ by latter method.

\section{Example}

In this section, to explain proposed method, an example is illustrated. To order six alternatives ( $n=6 ; a_{1}$ to $a_{6}$ ), decision maker selects five criteria ( $m=5 ; c_{1}$ to $c_{5}$ ) and carefully sets the standard in each criterion.

For each standard, based on Table 1, we construct the matrix $D$, as follows: 


$$
D=\left[\begin{array}{llllll}
2 & 2 & 0 & 1 & 1 & 0 \\
2 & 1 & 2 & 2 & 2 & 2 \\
2 & 2 & 1 & 1 & 1 & 2 \\
0 & 0 & 0 & 2 & 2 & 0 \\
2 & 2 & 2 & 0 & 2 & 2
\end{array}\right] .
$$

For example, $d_{13}=0$, the element of the matrix $D$, means alternative $a_{3}$ not satisfy the standard of criterion $c_{1}$.

\subsection{Example in AHP}

Following proposed procedure (W-0) to (W-5), described in section 2.1, we construct comparison matrix $A c_{1}$ to $A c_{5}$ based on $D$ and construct $W$. For example, with respect to criterion $c_{1}$, we use $d_{1 j}, 1 s t$-row vector of $D$, as follows:

$$
d_{1 j}=\left[\begin{array}{llllll}
2 & 2 & 0 & 1 & 1 & 0
\end{array}\right] .
$$

Based on (6), we have comparison matrix $A c_{1}$, as follows:

$$
A c_{1}=\left[\begin{array}{cccccc}
1 & 1 & \theta & \theta & \theta & \theta \\
1 & 1 & \theta & \theta & \theta & \theta \\
1 / \theta & 1 / \theta & 1 & 1 / \theta & 1 / \theta & 1 \\
1 / \theta & 1 / \theta & \theta & 1 & 1 & \theta \\
1 / \theta & 1 / \theta & \theta & 1 & 1 & \theta \\
1 / \theta & 1 / \theta & 1 & 1 / \theta & 1 / \theta & 1
\end{array}\right]
$$

Next, from (7), we calculate $W c_{1}$, principle eigen vector of $A c_{1}$, by power method where $\theta=2$. The convergence limit in this method is $10^{-6}$. The result of $W c_{1}$ is shown as (8).

$$
W c_{1}=\left[\begin{array}{l}
0.24669 \\
0.24669 \\
0.09790 \\
0.15540 \\
0.15540 \\
0.09790
\end{array}\right]
$$

As similar to have $W c_{1}$, we also have $W c_{2}$ to $W c_{5}$ and construct matrix $W$. The result of $W$ is shown as below:

$$
W=\left[\begin{array}{lllll}
0.24669 & 0.18181 & 0.22222 & 0.12500 & 0.18181 \\
0.24669 & 0.09090 & 0.22222 & 0.12500 & 0.18181 \\
0.09790 & 0.18181 & 0.11111 & 0.12500 & 0.18181 \\
0.15540 & 0.18181 & 0.11111 & 0.25000 & 0.09090 \\
0.15540 & 0.18181 & 0.11111 & 0.25000 & 0.18181 \\
0.09790 & 0.18181 & 0.22222 & 0.12500 & 0.18181
\end{array}\right] .
$$


Next, we calculate $V$ based on frequency of evaluation value. Frequency of evaluation value, in this example, is shown in Table 2.

Table 2 Frequency of Evaluation Value

\begin{tabular}{cccc}
\hline $\begin{array}{c}\text { criterion } \backslash \text { valu } \\
\mathrm{e}\end{array}$ & 2 & 1 & 0 \\
\hline$c_{1}$ & 2 & 2 & 2 \\
$c_{2}$ & 5 & 1 & 0 \\
$c_{3}$ & 3 & 3 & 0 \\
$c_{4}$ & 2 & 0 & 4 \\
$c_{5}$ & 5 & 0 & 1 \\
\hline
\end{tabular}

In this study, we calculate infrequent case and frequent case, respectively. First, we calculate $\boldsymbol{V}$ based on infrequent of satisfy the standard of each criterion. From Table 2, we have the infrequent order of criteria, $c_{4}>c_{1}>c_{3}>c_{5}>c_{2}$. Based on above order, we construct binary comparison matrix $V_{l}$, as follows:

$$
V_{l}=\left[\begin{array}{ccccc}
1 & \theta & \theta & 1 / \theta & \theta \\
1 / \theta & 1 & 1 / \theta & 1 / \theta & 1 / \theta \\
1 / \theta & \theta & 1 & 1 / \theta & \theta \\
\theta & \theta & \theta & 1 & \theta \\
1 / \theta & \theta & 1 / \theta & 1 / \theta & 1
\end{array}\right] .
$$

From (10), where $\theta=2$, we have $v_{l}$, as follows:

$$
v_{l}=\left[\begin{array}{l}
0.244679 \\
0.106503 \\
0.185432 \\
0.322856 \\
0.140531
\end{array}\right] .
$$

From (1), (9) and (11), we can obtain $w_{l}$, as follows:

$$
w_{l}=\left[\begin{array}{l}
0.187332 \\
0.175436 \\
0.133652 \\
0.166759 \\
0.182456 \\
0.154364
\end{array}\right] .
$$

From (12), as a result in infrequent case, we have the order of alternatives, $a_{1}>a_{5}>a_{2}>a_{4}>a_{6}>a_{3}$.

On the other hand, we have the order of criteria in frequent, $c_{2}>c_{5}>c_{3}>c_{1}>c_{4}$. As similar to infrequent case, we have $v_{h}$ and $w_{h}$, as follows: 


$$
\begin{gathered}
v_{h}=\left[\begin{array}{l}
0.140531 \\
0.322856 \\
0.185432 \\
0.106503 \\
0.244679
\end{array}\right], \\
w_{h}=\left[\begin{array}{l}
0.191202 \\
0.158601 \\
0.155360 \\
0.148023 \\
0.172730 \\
0.174084
\end{array}\right] .
\end{gathered}
$$

From (14), as a result in frequent case, we have the order of alternatives, $a_{1}>a_{6}>a_{5}>a_{2}>a_{3}>a_{4}$.

The result, the order of criterion and alternatives in infrequent case and frequent case are summarized in Table 3.

Table 3 The order of criterion and alternatives in AHP

\begin{tabular}{lllllllllllll}
\hline & \multicolumn{1}{c}{ The order of criterion } & \multicolumn{1}{c}{ The order of alternative } \\
\hline Infrequent & $c_{4}$ & $c_{1}$ & $c_{3}$ & $c_{5}$ & $c_{2}$ & & $a_{1}$ & $a_{5}$ & $a_{2}$ & $a_{4}$ & $a_{6}$ & $a_{3}$ \\
Frequent & $c_{2}$ & $c_{5}$ & $c_{3}$ & $c_{1}$ & $c_{4}$ & & $a_{1}$ & $a_{6}$ & $a_{5}$ & $a_{2}$ & $a_{3}$ & $a_{4}$ \\
\hline
\end{tabular}

In Table 3, the order of alternative $a_{1}$ is high even if the order of criteria is different.

\subsection{Example in 2-cluster-ANP}

Following proposed procedure (V-0) to (V-5), described in section 2.2, we construct comparison matrix $A a_{1}$ to $A a_{6}$ based on $D$ and construct $V$. For example $A a_{1}$, we use $d^{T}{ }_{i 1}, 1 s t$-column vector of $D$, as follows:

$$
d^{T}{ }_{i 1}=\left[\begin{array}{lllll}
2 & 2 & 2 & 0 & 2
\end{array}\right] .
$$

Based on (15), we have comparison matrix $A a_{1}$, as follows:

$$
A a_{1}=\left[\begin{array}{ccccc}
1 & 1 & 1 & \theta & 1 \\
1 & 1 & 1 & \theta & 1 \\
1 & 1 & 1 & \theta & 1 \\
1 / \theta & 1 / \theta & 1 / \theta & 1 & 1 / \theta \\
1 & 1 & 1 & \theta & 1
\end{array}\right]
$$

From (16), we have $V a_{1}$ where $\theta=2$, as follows: 


$$
V a_{1}=\left[\begin{array}{l}
0.222222 \\
0.222222 \\
0.222222 \\
0.111111 \\
0.222222
\end{array}\right]
$$

As similar to construct $W$, we have $V a_{2}$ to $V a_{6}$ and construct matrix $V$, as follows:

$$
V=\left[\begin{array}{llllll}
0.222222 & 0.248182 & 0.122622 & 0.162803 & 0.125000 & 0.125000 \\
0.222222 & 0.145773 & 0.282583 & 0.282583 & 0.250000 & 0.250000 \\
0.222222 & 0.248182 & 0.189591 & 0.162803 & 0.125000 & 0.250000 \\
0.111111 & 0.109682 & 0.122622 & 0.282583 & 0.250000 & 0.125000 \\
0.222222 & 0.248182 & 0.282583 & 0.109228 & 0.250000 & 0.250000
\end{array}\right] .
$$

As a result, we have super matrix $S$ of this example, as follows:

$S=\left[\begin{array}{ccccccccccc}0 & 0 & 0 & 0 & 0 & 0.22222 & 0.24818 & 0.12262 & 0.16280 & 0.12500 & 0.12500 \\ 0 & 0 & 0 & 0 & 0 & 0.22222 & 0.14577 & 0.28258 & 0.28258 & 0.25000 & 0.25000 \\ 0 & 0 & 0 & 0 & 0 & 0.22222 & 0.24818 & 0.18959 & 0.16280 & 0.12500 & 0.25000 \\ 0 & 0 & 0 & 0 & 0 & 0.11111 & 0.10968 & 0.12262 & 0.28258 & 0.25000 & 0.12500 \\ 0 & 0 & 0 & 0 & 0 & 0.22222 & 0.24818 & 0.28258 & 0.10922 & 0.25000 & 0.25000 \\ 0.24669 & 0.18181 & 0.22222 & 0.12500 & 0.18181 & 0 & 0 & 0 & 0 & 0 & 0 \\ 0.24669 & 0.09090 & 0.22222 & 0.12500 & 0.18181 & 0 & 0 & 0 & 0 & 0 & 0 \\ 0.09790 & 0.18181 & 0.11111 & 0.12500 & 0.18181 & 0 & 0 & 0 & 0 & 0 & 0 \\ 0.15540 & 0.18181 & 0.11111 & 0.25000 & 0.09090 & 0 & 0 & 0 & 0 & 0 & 0 \\ 0.15540 & 0.18181 & 0.11111 & 0.25000 & 0.18181 & 0 & 0 & 0 & 0 & 0 & 0 \\ 0.09790 & 0.18181 & 0.22222 & 0.12500 & 0.18181 & 0 & 0 & 0 & 0 & 0 & 0\end{array}\right]$

To calculate $w$ and $v$ from (19), we calculate the principle eigen vector corresponding to the eigen value 1 .

As a result, normalizing the sum of weights equal to one, we have (20) and (21), as follows:

$$
v=\left[\begin{array}{l}
0.170038 \\
0.236658 \\
0.200461 \\
0.165426 \\
0.227416
\end{array}\right]
$$




$$
w=\left[\begin{array}{l}
0.191550 \\
0.170035 \\
0.143976 \\
0.153758 \\
0.174432 \\
0.166249
\end{array}\right]
$$

Then we have the order of criteria, $c_{2}>c_{5}>c_{3}>c_{1}>c_{4}$. And we have the order of alternatives, $a_{1}>a_{5}>$ $a_{2}>a_{6}>a_{4}>a_{3}$.

Next, the results, the order of alternative for various values of $\theta$, are shown in Table 4 .

Table 4 The order of alternative for various values of $\theta$

\begin{tabular}{cccccccc}
\hline$\theta$ & \multicolumn{1}{c}{ The order of alternative } \\
\hline 2 & $a_{1}$ & $a_{5}$ & $a_{2}$ & $a_{6}$ & $a_{4}$ & $a_{3}$ \\
4 & $a_{1}$ & $a_{5}$ & $a_{2}$ & $a_{6}$ & $a_{4}$ & $a_{3}$ \\
8 & $a_{1}$ & $a_{5}$ & $a_{2}$ & $a_{6}$ & $a_{4}$ & $a_{3}$ \\
16 & $a_{1}$ & $a_{5}$ & $a_{6}$ & $a_{2}$ & $a_{4}$ & $a_{3}$ \\
32 & & $a_{1}$ & $a_{5}$ & $a_{6}$ & $a_{2}$ & $a_{4}$ & $a_{3}$ \\
64 & $a_{1}$ & $a_{5}$ & $a_{6}$ & $a_{2}$ & $a_{4}$ & $a_{3}$ \\
128 & $a_{1}$ & $a_{5}$ & $a_{6}$ & $a_{2}$ & $a_{4}$ & $a_{3}$ \\
512 & $a_{1}$ & $a_{5}$ & $a_{6}$ & $a_{2}$ & $a_{4}$ & $a_{3}$ \\
\hline
\end{tabular}

The result of $\theta=8$ or above, in Table $4, a_{2}$ and $a_{6}$ reverse the order. However, we do not know suitable value of $\theta$. Moreover the order of criterion and alternatives in ANP and AHP, where $\theta=2$, are summarized in Table 5.

Table 5 The order of criterion and alternatives in ANP and AHP

\begin{tabular}{cccccccccccccc}
\hline & \multicolumn{1}{c}{ The order of criterion } & \multicolumn{1}{c}{ The order of alternative } \\
\hline ANP & $c_{2}$ & $c_{5}$ & $c_{3}$ & $c_{1}$ & $c_{4}$ & $a_{1}$ & $a_{5}$ & $a_{2}$ & $a_{6}$ & $a_{4}$ & $a_{3}$ \\
AHP & $c_{2}$ & $c_{5}$ & $c_{3}$ & $c_{1}$ & $c_{4}$ & $a_{1}$ & $a_{6}$ & $a_{5}$ & $a_{2}$ & $a_{3}$ & $a_{4}$ \\
$\begin{array}{c}\text { (frequent) } \\
\text { AHP } \\
\text { (infrequent) }\end{array}$ & $c_{4}$ & $c_{1}$ & $c_{3}$ & $c_{5}$ & $c_{2}$ & & $a_{1}$ & $a_{5}$ & $a_{2}$ & $a_{4}$ & $a_{6}$ & $a_{3}$ \\
\hline
\end{tabular}

In Table 5, the result of the order of criterion in ANP coincide with AHP in frequent case, however, the value of weights are not coincide.

\section{Conclusion}

In this paper, a method to speedily construct comparison matrix and super matrix was proposed and applied to example of AHP and 2-cluster ANP. By preparing each standard of criterion, comparison matrix and super matrix are constructing automatically and have weights immediately. As a result, by our method, it is possible to implement AHP in decision-making part of several software. In future, we need more discussion for accuracy of binary comparisons. 


\section{References}

Saaty, T. L. (1980) “The Analytic Hierarchy Process”, McGrawHill, NewYork.

Saaty, T. L. (2001) “The Analytic Network Process”, RWS Publications.

Takahashi, I. (1990) "AHP Applied to Binary and Ternary Comparisons", Journal of Operations Research Society of Japan, Vol. 33, No. 3, 199-206. 